\title{
A new implementation of narrowband interference detection, characterization, and mitigation technique for a software-defined multi-GNSS receiver
}

\author{
Nunzia Giorgia Ferrara ${ }^{1}$. Mohammad Zahidul H. Bhuiyan ${ }^{1}$. Stefan Söderholm ${ }^{1}$ - Laura Ruotsalainen ${ }^{1}$. \\ Heidi Kuusniemi ${ }^{1}$
}

Received: 23 November 2017 / Accepted: 23 July 2018 / Published online: 2 August 2018

(c) The Author(s) 2018

\begin{abstract}
Due to the very low power of satellite signals when reaching the earth's surface, global navigation satellite system receivers are vulnerable to various types of radio frequency interference, and, therefore, countermeasures are necessary. In the case of a narrowband interference (NBI), the adaptive notch filtering technique has been extensively investigated. However, the research on the topic has focused on the adaptation of the notch frequency, but not of the notch width. We present a fully adaptive solution to counter NBI. The technique is capable of detecting and characterizing any number of narrow interfered bands, and then optimizing the mitigation process based on such characterization, namely the estimates of both interference frequency and width. Its full adaptiveness makes it suitable to cope with the unpredictable and diverse nature of unintentional interfering events. In addition to a thorough performance evaluation of the proposed method, which shows its benefits in terms of signal quality improvement, an analysis of the impact of different NBI profiles on GPS L1 C/A and Galileo E1 is also conducted.
\end{abstract}

Keywords Software-defined multi-GNSS receiver $\cdot$ Narrowband interference detection and mitigation $\cdot$ Notch filter

\section{Introduction}

Global navigation satellite system (GNSS) signals are received at the earth's surface with very low power levels and are, therefore, susceptible to radio frequency interference (RFI) which may be caused unintentionally or intentionally. Unintentional sources of RFI include harmonic emissions from commercial high-power transmitters, ultrawideband radar, television, VHF, mobile satellite services, and personal electronic devices. The malicious intentional interference is instead produced by jammers, which aim at disrupting the receiver operation by deliberately broadcasting strong signals in the GNSS frequency band. RFI can also be classified as either narrowband or wideband, depending on the ratio between its bandwidth and the desired GNSS

Nunzia Giorgia Ferrara

giorgia.ferrara@nls.fi

1 Department of Navigation and Positioning, Finnish Geospatial Research Institute, National Land Survey, Geodeetinrinne 2, 02430 Kirkkonummi, Finland signal bandwidth. In general, interference can be considered as narrowband if its bandwidth is much less than $1 \mathrm{MHz}$ (Parkinson et al. 1996).

The presence of undesired signals in the GNSS band adversely affects the receiver performance. An assessment of the impact of different types of unintentional interference on the acquisition and tracking performance can be found in Wildemeersch et al. (2010). It is shown that, for the acquisition, the probability of detection and the probability of false alarm are vulnerable to the presence of interference, and that, for the tracking, the carrier-to-noise density ratio $\left(C / N_{0}\right)$ decreases and the variance of the position solution increases. In addition to $C / N_{0}$, other observables available at different stages of the receiver processing chain are affected by the jammer signal (Borio et al. 2016). For example, the automatic gain control (AGC) level drops in response to increased power in the GNSS band, and the running delay lock loop (DLL) variance and the running phase lock loop (PLL) variance increase due to the rise in the noise level (Bhuiyan et al. 2014a). In general, the interference causes degradation in terms of raw data availability and reliability and consequently a less accurate position, velocity, and time 
(PVT) solution, and in the worst case, it can cause a complete denial of the service (Kuusniemi et al. 2012).

Therefore, in order to provide good positioning services, the receiver should be able to identify an interference occurrence and mitigate its effects. We focus on narrowband interference (NBI) affecting GPS L1 C/A and Galileo E1 signals. For this type of interference, the adaptive notch filtering has been extensively used as a mitigation technique (Raasakka and Orejas 2014; Borio et al. 2006). However, the typical approach is to adjust the notch frequency according to the estimated interference frequency, without adapting the notch width, namely the width of the frequency interval where the signal power is attenuated. We present, instead, a fully adaptive implementation of NBI detection, characterization and mitigation technique which utilizes the estimation of the interference bandwidth to also adapt the notch width, in order to optimize the capability of attenuating the interference while preserving the useful GNSS signal. This full adaptiveness makes the proposed approach capable of dealing with any number of narrow interfered bands with unknown center frequencies and unknown widths.

After a brief overview of the existing interference detection and mitigation techniques, we describe the new countermeasure against NBI in GNSS. The technique is validated with a multi-GNSS software-defined receiver, and results show that it is capable of efficiently detecting, characterizing, and mitigating the interference.

\section{Interference detection}

In order to mitigate the effects of interference from intentional or unintentional sources, reliable interference detection must be conducted first. The interference detection techniques can be broadly classified into three main categories depending on which stage of the receiver processing chain the technique is being implemented: (1) detection at hardware stage based on AGC output analysis, (2) at digital signal processing stage based on digitized intermediate frequency (IF) data analysis, and (3) at post-correlation stage based on correlator measurements/signal-to-noise power ratio, etc. (Borio et al. 2016).

In the presence of any kind of interference at the signal band of interest, the AGC needs to reduce its gain to be able to minimize potential quantization errors. The impact of various types of interference on AGC circuit was analyzed in many research articles, for example, in Axell et al. (2015) and Lindstrom et al. (2007), and it was concluded that the AGC can be well utilized as an interference assessment tool for GNSS receivers.

Digital signal processing based techniques generally operate on the digitized signal samples at the output of the $\mathrm{RF}$ front-end. Any interfering signal intruding the antenna with the power level exceeding the noise floor is expected to be detectable via signal spectrum analysis. This can be achieved by comparing the estimated power spectral density (PSD) of the received signal with that of a spectral mask in a nominal interference-free condition (Balaei and Dempster 2009). Different interference detection approaches can also be adopted based on the stream of raw digitized samples in the time domain. The main idea behind the time domain approaches is that the digitized RF samples at the output of ADC closely follow a Gaussian distribution in nominal interference-free condition. Therefore, interference detection methods can be implemented by detecting deviations from the Gaussian distribution. Examples of techniques based on the time domain processing of the statistical characteristics of the RF samples can be found in Bhuiyan et al. (2014a) and Motella and Lo Presti (2014).

Finally, interference detection techniques at the post-correlation stage exploit the observables provided by a GNSS receiver after the correlation process (Sheridan et al. 2012). Such receiver observables are correlator output power, such as DLL or PLL discriminator outputs, variance of correlator output power, carrier phase vacillation, $C / N_{0}$, etc. Among all of them, $C / N_{0}$-based detection is the most popularly used method (Calcagno et al. 2010).

\section{Interference mitigation}

Interference mitigation techniques can be divided into four categories: (1) techniques based on signal processing, (2) antenna configuration, (3) sensor integration, and (4) system deployment.

Traditionally, interference mitigation techniques have mainly been based on signal processing means. By transforming the signal into a new domain, the useful component and interference may be separated (Dovis et al. 2012). Several examples of transformed domain techniques can be found in literature, such as those exploiting the Wavelet transform (Musumeci and Dovis 2014), the Hilbert-Huang transform (Fadaei et al. 2015), and the Karhunen-Loéve transform (Dovis and Musumeci 2016). Although these methods show good performance in mitigating the interference, their implementation is computationally heavy. Notch filtering, where a deep null is placed in correspondence of the interference frequency, is a more computationally efficient solution.

Antenna arrays may be used for mitigating both narrowband and broadband interference signals. An example can be found in Daneshmand et al. (2013), where a two-stage beam-former utilizing an antenna array was developed to mitigate multipath as well as interference.

One of the most promising techniques for enhancing GNSS robustness against interference is the use of deeply 
coupled integration of self-contained sensors into the GNSS receiver. The measurements obtained using sensors are not affected by interference and, therefore, the integration results in a system with enhanced robustness. However, inertial navigation system (INS) measurements suffer from biases that accumulate with time. Visual sensors, i.e., cameras, are feasible instruments for constricting the growth of the errors and are resistant to GNSS jamming. In favorable environments and special camera configuration, camera attitude and translation between consecutive images may be detected (Ruotsalainen et al. 2012). These measurements may be used to form a deeply coupled GNSS/INS/visual sensor integration that will further improve the robustness of the positioning accuracy in situations where the interference is not only momentary (Ruotsalainen et al. 2014).

Finally, it is very rare that one jammer could interfere with signals from multiple systems having different frequencies. Therefore, the use of multi-GNSS, namely of a receiver that is capable of flexibly changing the frequency band of signals processed from one that is being interfered to another not being disrupted, can provide interference mitigation capability. However, use of multiple frequency bands requires increased processing power from the receiver, and, therefore, the receiver implementation should be carefully considered.

\section{New narrowband interference countermeasure}

This section presents a new method to detect, characterize, and mitigate narrowband interfering signals. The technique is implemented at the digital signal processing stage of the receiver, and it is capable of identifying an unknown number of narrow interfered bands in the GNSS spectrum. Once the interferences have been detected, the mitigation process is optimized based on their characterization, i.e., the estimates of their central frequency and their width.

NBI can be generated unintentionally by radio devices which operate close to the GNSS frequencies or at their subharmonics (Sánchez-Naranjo et al. 2017). Due to the unpredictable nature of these events and to the variety of potential interference sources, the number of interfered bands in the GNSS spectrum, as well as their central frequencies and their widths, can be different. Examples of such diversity are given in Fig. 1, which shows some real-life NBI events detected by the detector probe, which is an interference detection unit developed by Nottingham Scientific Limited (NSL). The detector probes are deployed in many different locations of the world within the H2020 STRIKE3 project (STRIKE3 2016) that aims to monitor the international GNSS threat scene to capture the scale and dynamics of the problem and to cooperate with international GNSS partners to develop, negotiate, promote, and implement standards for threat reporting and receiver testing (Thombre et al. 2017). The NBI events presented in Fig. 1 are one of most common event types for the detector probe located near an airport in Finland. These types of NBI events are detected on a daily basis, and they disrupt the protected L1/E1 GNSS frequency band.

The following subsections describe how the proposed technique is able to first estimate the central frequencies and the bandwidths of any number of NBI, and then to use these estimates to optimize the mitigation scheme.

\section{Proposed interference detection and characterization}

The interference detection and estimation are implemented in the frequency domain. More specifically, the PSD of the digitized received signal at the output of the RF front-end is estimated via simple fast Fourier transform (FFT) method. The signal samples are processed in blocks of $T_{\text {coh }}$ length, with $T_{\text {coh }}$ being the coherent integration time. In a nominal interference-free condition, GNSS signals are buried under the noise level, as shown in the top panel of Fig. 2, whereas the interferences are much stronger. Therefore, their presence in the GNSS band causes alterations in the spectrum, as depicted in the bottom panel of Fig. 2.

The proposed interference detection and estimation strategy identifies and characterizes such alterations by carrying out the following steps:

Step 1 The PSD of the received incoming signal is estimated via FFT.
Fig. 1 Examples of few frequently occurring real NBI events at a host site in Finland. Courtesy to NSL
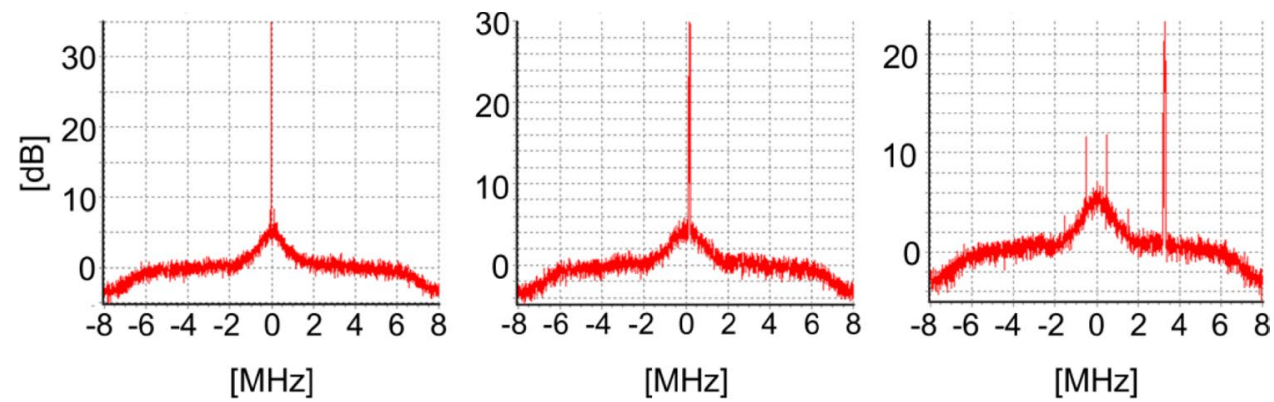

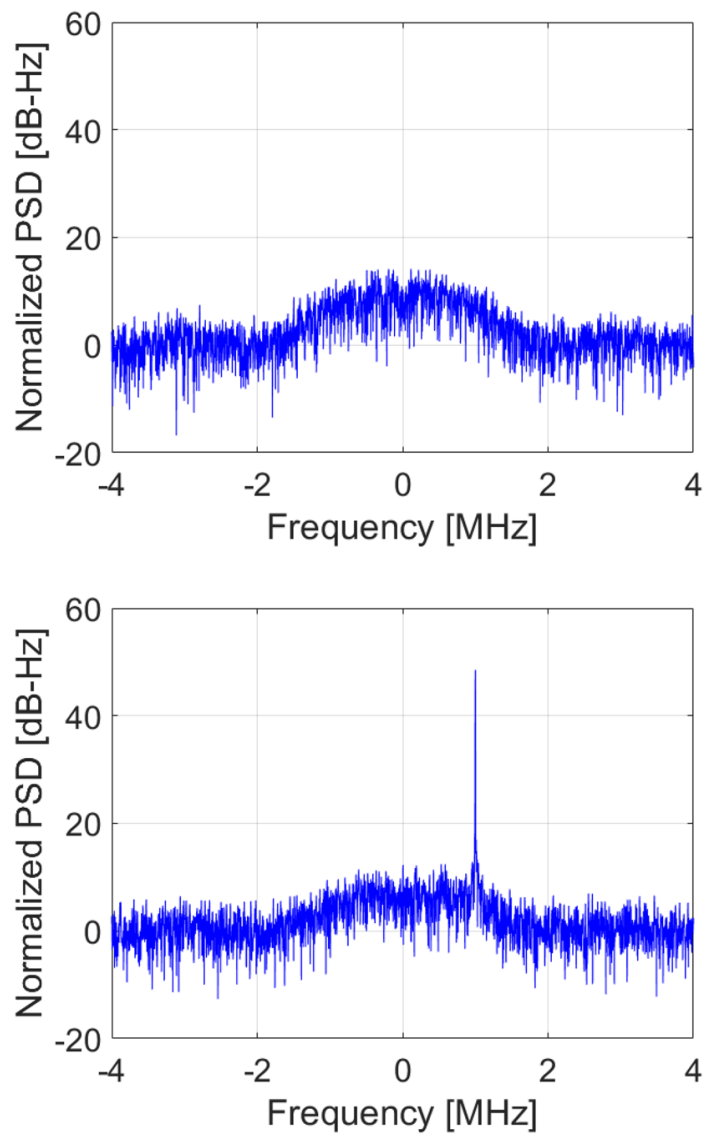

Fig. 2 PSD of an interference-free GNSS signal (top) and of a GNSS signal affected by NBI (bottom)

Step 2 Each PSD value is compared to a threshold in order to identify the spectral components that belong to bands affected by interference. The threshold $T$ is set based on the mean, $\mu_{\mathrm{PSD}}$, and the standard deviation, $\sigma_{\mathrm{PSD}}$, of the PSD values. In particular, a spectral component is considered to belong to an interfered band if its corresponding PSD value is higher than the mean by more than three standard deviations. In other words, $T$ is equal to $\mu_{\mathrm{PSD}}+3 \cdot \sigma_{\mathrm{PSD}}$.

Step 3 An auxiliary vector is then built to estimate the central frequency and the bandwidth of the interfered bands. In correspondence of the anomalous PSD values, i.e., the ones above the threshold, the auxiliary vector is set to 1 ; otherwise to 0 .

Step 4 The width and the central frequency of each interfered band are then derived from the groups of interfered frequencies, namely the ones at which the auxiliary vector is equal to 1 . If consecutive interfered portions of the spectrum are less than $10 \mathrm{kHz}$ apart from each other, they are considered as belonging to the same interfered band and grouped together. Furthermore, the minimum bandwidth that the algorithm is designed to consider for a NBI is set to $3 \mathrm{kHz}$.
The top panel of Fig. 3 shows the spectrum of a GPS signal affected by two narrowband interferences at $f_{\mathrm{L} 1 / \mathrm{E} 1} \pm 0.5 \mathrm{MHz}$, with $f_{\mathrm{L} 1 / \mathrm{E} 1}$ being the L1/E1 carrier frequency $\left(f_{\mathrm{L} 1 / \mathrm{E} 1}=1575.42 \mathrm{MHz}\right)$. The interval $f_{\mathrm{L} 1 / \mathrm{E} 1} \pm 2 \mathrm{MHz}$ only is shown. The light-blue line represents the mean of the PSD values, while the green line represents the threshold used to identify the interfered bands. The anomalous PSD values are depicted in red. The auxiliary vector is shown in the bottom panel of Fig. 3. In correspondence of the anomalous PSD values, the auxiliary vector is 1 ; otherwise it is 0 .

The NBI detection/characterization process is shown schematically in Fig. 4. The output of this block is a pair of vectors, $\boldsymbol{B} \boldsymbol{w}=\left[b w_{1}, b w_{2}, \ldots, b w_{N}\right]$ and $\boldsymbol{F}=\left[f_{1}, f_{2}, \ldots, f_{N}\right]$, which contain, respectively, the bandwidths and the center frequencies of the $N$ identified interfered bands.

\section{Proposed interference mitigation}

If at least one interference has been detected, namely $\boldsymbol{B} \boldsymbol{w}$ and $\boldsymbol{F}$ are not empty vectors, mitigation is performed by applying a one-pole notch filter for each identified interfered band. Each filter is configured according to the output of the interference detection/estimation unit. A one-pole notch filter has a zero in $z_{0}=\alpha e^{-j 2 \pi f_{i}}$ corresponding to the center frequency $f_{i}$ of the $i$-th interfered band to be removed, and a pole in $z=k z_{0}$ to compensate for the effects of the zero, where the parameter $k$, which has to be less than 1 for stability reasons, is the pole contraction factor and regulates the notch width. The closer $k$ is to unity, the narrower the width of the notch (Borio et al. 2006). The filter, whose transfer function is given by

$H(z)=\frac{1-z_{0} z^{-1}}{1-k z_{0} z^{-1}}$

is, therefore, fully characterized by two parameters: the notch frequency and the notch width.

The notch filter has been extensively used as a mitigation technique against NBI in GNSS (Raasakka and Orejas 2014; Borio et al. 2006). However, the typical approach is to use an adaptive notch filter which is capable of tracking the interference frequency and adjusting the notch frequency accordingly but does not adapt the notch width. We propose, instead, a technique which utilizes the estimation of the interference bandwidth to also adapt the parameter $k$, and hence the notch width, in order to optimize the filter capability of attenuating the interference without excessively disrupting the useful GNSS signal. For each identified interfered band, the estimated frequency $\left(f_{i}, i=1,2, \ldots, N\right)$ is used as the notch frequency, and the estimated bandwidth $\left(b w_{i}, i=1,2, \ldots, N\right)$ is used to set $k_{i}$ as follows (Borio et al. 2006): 
Fig. 3 PSD of a GPS signal affected by two NBI (top) and auxiliary vector used to identify the interfered frequencies and the corresponding width (bottom)
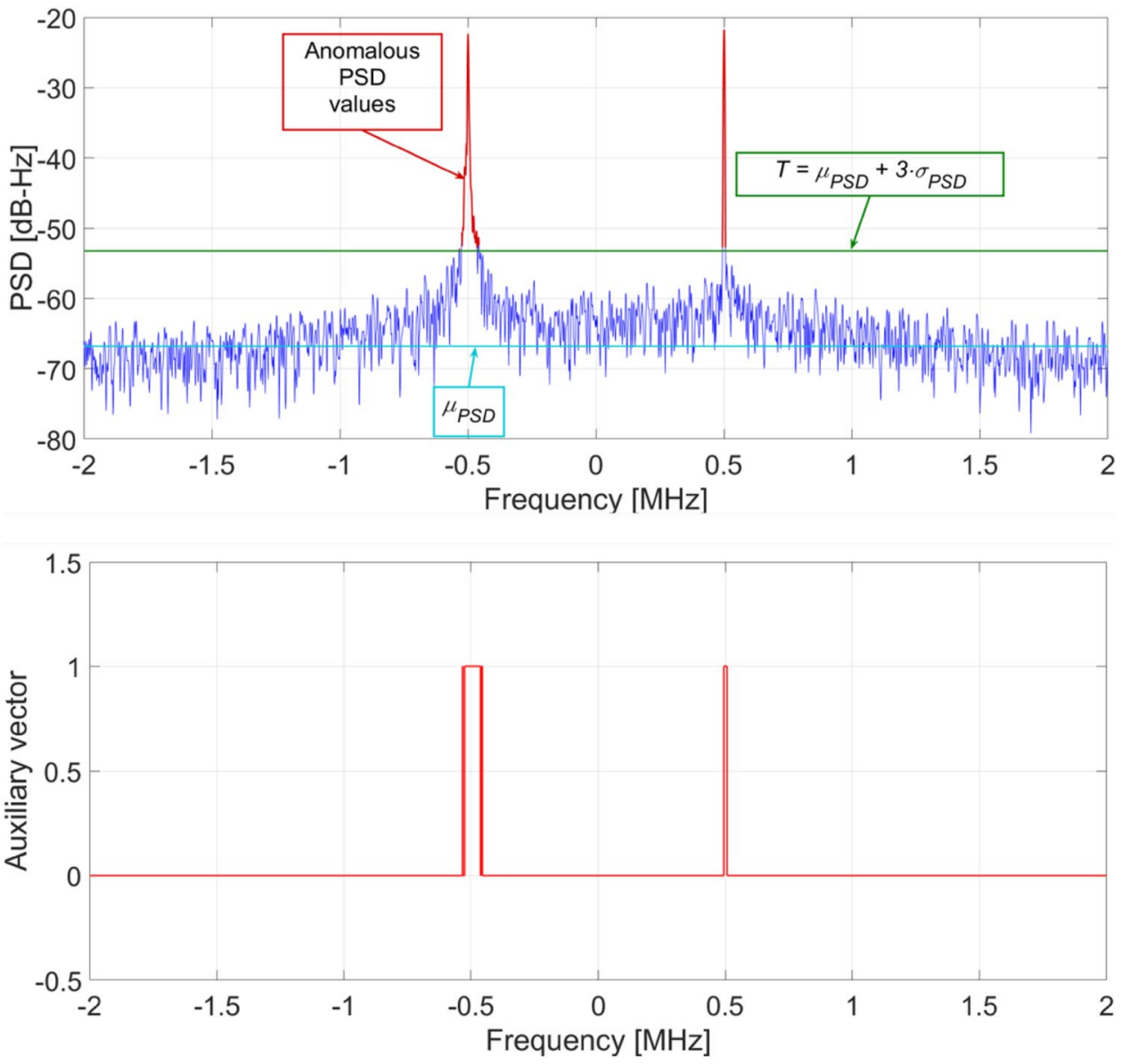

$k_{i}=f\left(b w_{i}\right)=1-\frac{\pi \cdot b w_{i}}{f_{\mathrm{s}}}$,

where $f_{\mathrm{s}}$ is the sampling frequency. This full adaptiveness makes the proposed approach capable of dealing with the unpredictable and diverse nature of unintentional NBI in GNSS spectrum. The adaptation rate is decided by the detection/estimation unit which processes the raw samples in blocks of $T_{\text {coh }}$ length. In other words, the pair $\{\boldsymbol{B} \boldsymbol{w}, \boldsymbol{F}\}$ at the output of the detection/estimation unit and, consequently, the number, center frequencies, and bandwidths of the notch filters applied in the mitigation unit are updated once every coherent integration period.

Figure 5 shows schematically how the mitigation is implemented, and an overview of the proposed NBI countermeasure is given in Fig. 6. The GNSS signal is filtered, down-converted to the IF, and digitized by the front-end. The interference detection and estimation unit then processes the IF samples in the frequency domain to decide if any interference is present. If so, the mitigation block uses the outputs of the detection/estimation unit ( $\boldsymbol{B} \boldsymbol{w}$ and $\boldsymbol{F})$ to optimally remove the interferences; otherwise, the
IF samples are passed directly to the receiver tracking block.

\section{Multi-GNSS software-defined receiver as a validation tool}

The Finnish Geospatial Research Institute (FGI) developed an in-house software-defined multi-constellation, multi-frequency GNSS receiver called FGI-GSRx, which is capable of processing signals from GPS, GLONASS, Galileo, BeiDou, and the Indian Regional Navigation Satellite System. The FGI-GSRx has support for a number of different RF front-ends. It is completely configurable, which makes it suitable for any sort of algorithm development and performance testing (Söderholm et al. 2016; Bhuiyan et al. 2014c; Thombre et al. 2015).

The performance of the implemented NBI countermeasure was evaluated with the FGI-GSRx at the tracking stage and at the navigation stage. Fundamental to assessing the status of GNSS receiver tracking operation, the measure of $C / N_{0}$ can be used to determine the effect of NBI on GNSS 


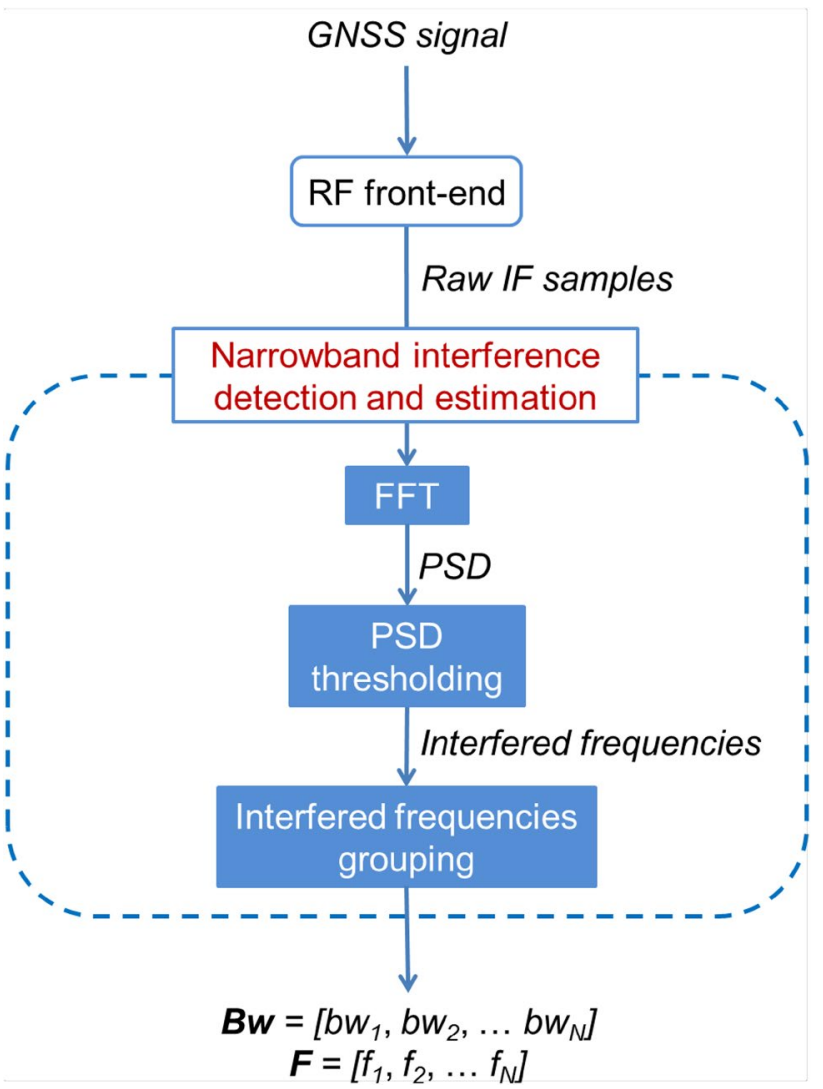

Fig. 4 NBI detection and estimation process

signals and to validate the mitigation algorithm against it. In this implementation, $C / N_{0}$ is estimated based on the signal-to-noise power ratio, where the signal power is estimated from the prompt correlation output, and the noise power is estimated from the output of the correlation with $a+2$ chips early locally generated code replica (Bhuiyan et al. 2014b).

\section{Experimental set-up}

In order to assess the performance of the proposed NBI countermeasure, a set of tests was conducted using simulated GNSS signals. A multi-constellation hardware GNSS simulator, Spectracom GSG-6 Series, was used to generate GPS L1 C/A and Galileo E1 RF signals in a static receiver scenario under nominal open sky conditions. The simulator was also used to generate the interference in the L1/E1 band. In particular, four interference profiles were considered in the tests, as described in Table 1, and in all the cases the interfering signal was turned on about $45 \mathrm{~s}$ after the start of the data capture and then off after about 45-50 s. Each data capture lasted in total for $2 \mathrm{~min}$. The GNSS signal transmit

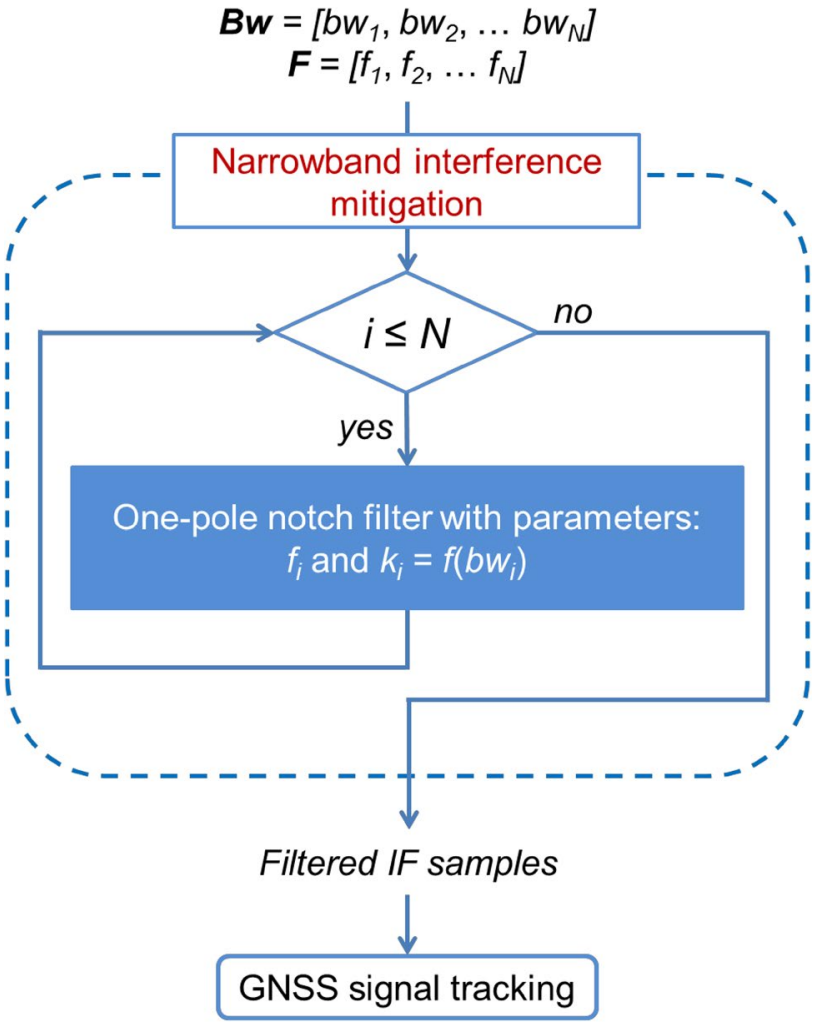

Fig. 5 NBI mitigation process

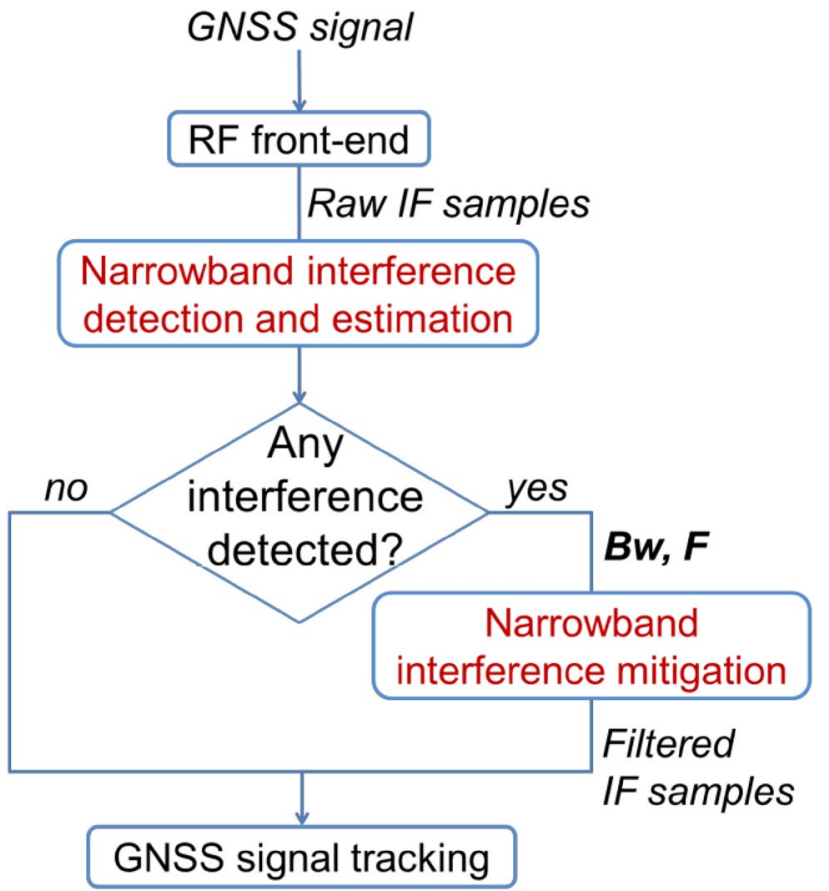

Fig. 6 Block diagram of the proposed NBI countermeasure 
Table 1 Tested interference profiles

\begin{tabular}{lll}
\hline Test case id & Interference type & $\begin{array}{l}\text { Interference } \\
\text { time interval } \\
(\mathrm{s})\end{array}$ \\
\hline $\mathrm{i}$ & $\mathrm{NBI}$ at $f_{\mathrm{L} 1 / \mathrm{E} 1}$ & $46-91$ \\
ii & $\mathrm{NBI}$ at $f_{\mathrm{L} 1 / \mathrm{E} 1}+1 \mathrm{MHz}$ & $47-90$ \\
iii & $\mathrm{NBI}$ at $f_{\mathrm{L} 1 / \mathrm{E} 1} \pm 1 \mathrm{MHz}$ & $51-100$ \\
iv & $\mathrm{NBI}$ at $f_{\mathrm{L} 1 / \mathrm{E} 1}$ and at & $47-97$ \\
& $f_{\mathrm{L} 1 / \mathrm{E} 1}+1 \mathrm{MHz}$ & \\
\hline
\end{tabular}

Table 2 Configuration for GTEC RF front-end

\begin{tabular}{ll}
\hline Parameter & Value \\
\hline Intermediate frequency (MHz) & 1574.891 \\
Bandwidth (MHz) & 18 \\
Sampling frequency (MHz) & 20.25 \\
Number of quantization bits & 16 \\
Received bands & GPS L1/Galileo E1 \\
\hline
\end{tabular}

power was set to $-100 \mathrm{dBm}$. It has to be noted, however, that the transmission power setting of the Spectracom simulator is valid for GPS L1 C/A, whereas the other signals may have an offset relative to that (Spectracom 2017). In particular, the simulated Galileo E1 signal has a power level $1.5 \mathrm{~dB}$ lower than the simulated GPS L1 C/A. Finally, the NBI power was set to $-73 \mathrm{dBm}$.

The RF signal generated by the GNSS simulator was captured with a TeleOrbit GTEC RF front-end developed by Fraunhofer Institute for Integrated Circuits IIS (2013). The front-end configuration used in the tests is shown in Table 2.

The obtained raw IF data samples were then processed with the FGI-GSRx. Through the use of a splitter, the RF signal from the GNSS simulator (GNSS signal+interference) was also fed to a Javad DELTA receiver, to verify the effect of the four considered interference profiles on GPS and Galileo in the L1/E1 band. The test set-up used to validate the proposed technique is shown in Fig. 7.

\section{Results analysis}

Three main categories of results are presented in this section. First, the impact of different interference profiles on GPS L1 C/A and Galileo E1 is analyzed in terms of $C / N_{0}$ loss. Then, the performance of the proposed NBI countermeasure is evaluated in terms of detection, estimation, and mitigation capability, and finally, a comparison between the implemented technique and the adaptive notch filter is made.
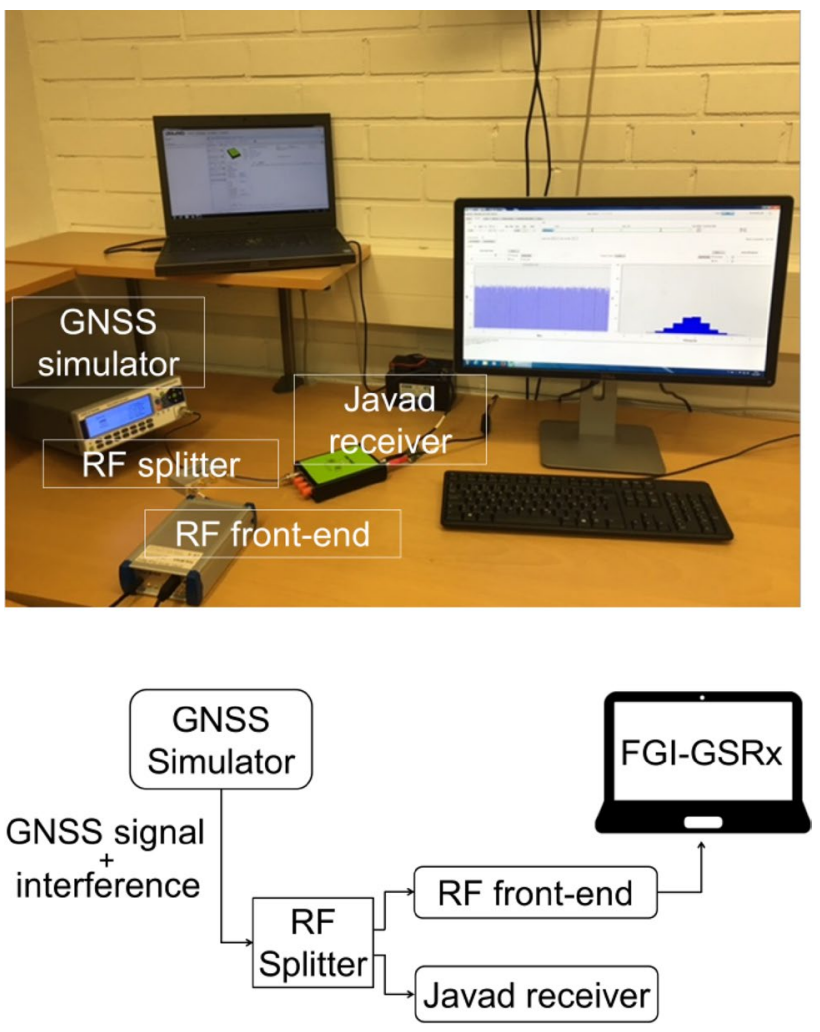

Fig. 7 Experimental set-up (top) and its schematic representation (bottom)

\section{Impact of different interference profiles on GPS and Galileo in the L1/E1 band}

Figure 8 shows the estimated average $C / N_{0}$ loss over time in different test cases. The average is computed among all the satellites in view for each navigation system. Each panel contains four curves: the two solid lines show the results obtained with the FGI-GSRx software-defined receiver for GPS (blue) and Galileo (orange), respectively, while the two dashed lines are obtained with the Javad receiver for GPS (blue) and Galileo (orange), respectively.

When the NBI is at $f_{\mathrm{L} 1 / \mathrm{E} 1}$, as in test case $\mathrm{i}$, GPS L1 C/A is highly affected, showing an average $C / N_{0}$ loss of about $15 \mathrm{~dB}$, whereas Galileo E1 suffers from only $3 \mathrm{~dB}$ degradation. This is mainly due to the different spectrum shape of the two signals. GPS L1 C/A utilizes binary phase shift key (BPSK) modulation and has, therefore, a sinc function shaped spectrum with a $2.046 \mathrm{MHz}$-wide main lobe around $f_{\mathrm{L} 1 / \mathrm{E} 1}$ (Global Positioning Systems Directorate 2015). Galileo E1 utilizes, instead, binary offset carrier (BOC) modulation, where, through the use of a sub-carrier, the spectrum of the signal is divided into two parts around $f_{\mathrm{L} 1 / \mathrm{E} 1}$ with two $2.046 \mathrm{MHz}$-wide main lobes centered at $f_{\mathrm{L} 1 / \mathrm{E} 1} \pm 1.023 \mathrm{MHz}$ (European Union 2016). While BPSK-modulated signals have then most of their energy concentrated around the 
Fig. 8 Average GPS and Galileo $C / N_{0}$ loss when no mitigation is applied for test case i (top left), test case ii (top right), test case iii (bottom left), and test case iv (bottom right)

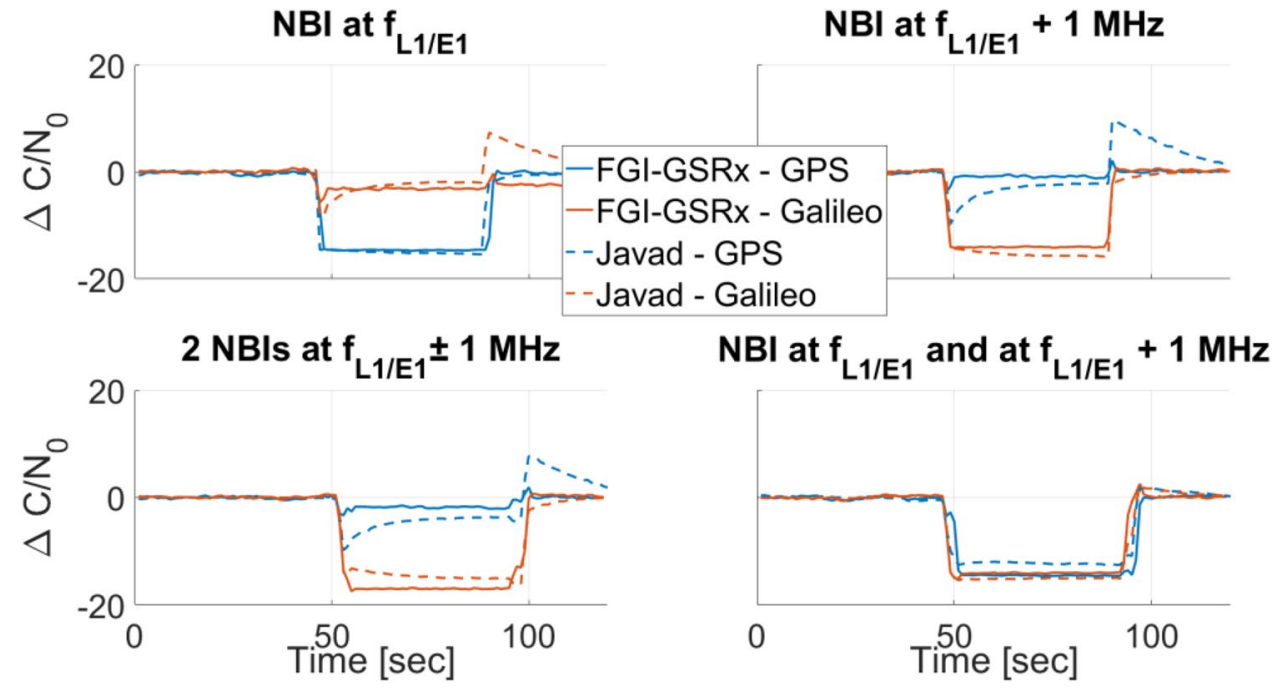

carrier frequency, the BOC-modulated signals have most of their energy concentrated around the centers of the two main lobes. For the same reason, when the NBI is $1 \mathrm{MHz}$ away from $f_{\mathrm{L} 1 / \mathrm{E} 1}$, as in test cases ii and iii, GPS L1 C/A is almost not affected (1-2 dB loss), while Galileo E1 is highly affected, showing a $C / N_{0}$ degradation of about $14 \mathrm{~dB}$ in the case of single NBI at $f_{\mathrm{L} 1 / \mathrm{E} 1}+1 \mathrm{MHz}$ and of about $17 \mathrm{~dB}$ when two NBI appear close to the center of both main lobes. Finally, when an NBI appears close to the center of GPS L1 C/A main lobe as well as close to the center of the Galileo E1 right main lobe, as in test case iv, the impact on the two signals is similar. For the signals that are not strongly affected by the interference, namely Galileo E1 in test case i, and GPS L1 C/A in test cases ii and iii, the Javad receiver's $C / N_{0}$ estimation seems to have a more abrupt degradation than the FGI-GSRx when the interference is turned on, and a spike when the interference is turned off. However, it seems to converge slowly to the right $C / N_{0}$ over time.

\section{Detection, estimation, and mitigation capability of the implemented approach}

The detection performance of the proposed algorithm was analyzed for three values of the threshold $T=\mu_{\mathrm{PSD}}+n_{\mathrm{std}} \cdot \sigma_{\mathrm{PSD}}$, with the number of standard deviations $n_{\text {std }}=2.5,2.8$, and 3. The detection unit output for test case $\mathrm{i}$ and for the three values of $T$ is shown in Fig. 9. If at least one interfered band has been detected, the detection output is equal to 1 and the mitigation unit is activated; otherwise, the output of the detection unit is equal to 0 and no mitigation is applied. The two vertical red lines indicate the interval during which the interference was on. It can be observed that the case $n_{\text {std }}=3$ gives the best performance, being capable of always detecting the interference when it is present and of generating no false alarms, namely the detection output is 1 when the interference is present and 0 when the interference is not present. Similar behavior was observed for the other interference profiles.
Fig. 9 Detection output for three values of $T=\mu_{\mathrm{PSD}}+n_{\mathrm{std}} \cdot \sigma_{\mathrm{PSD}}$ with $n_{\text {std }}=2.5$ (top), 2.8 (middle), and 3 (bottom)
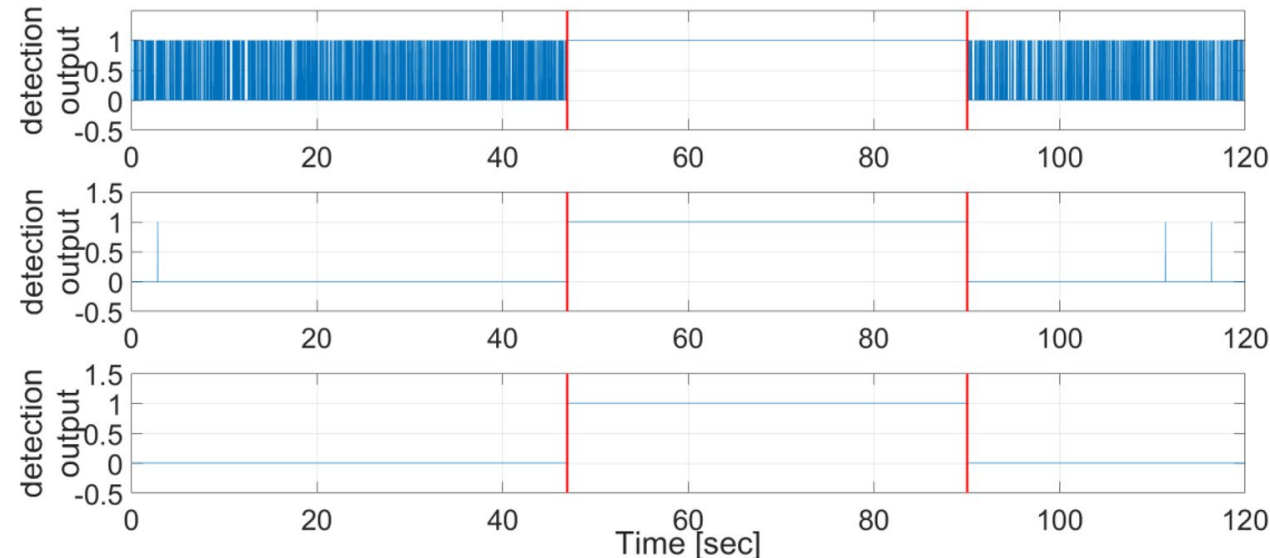

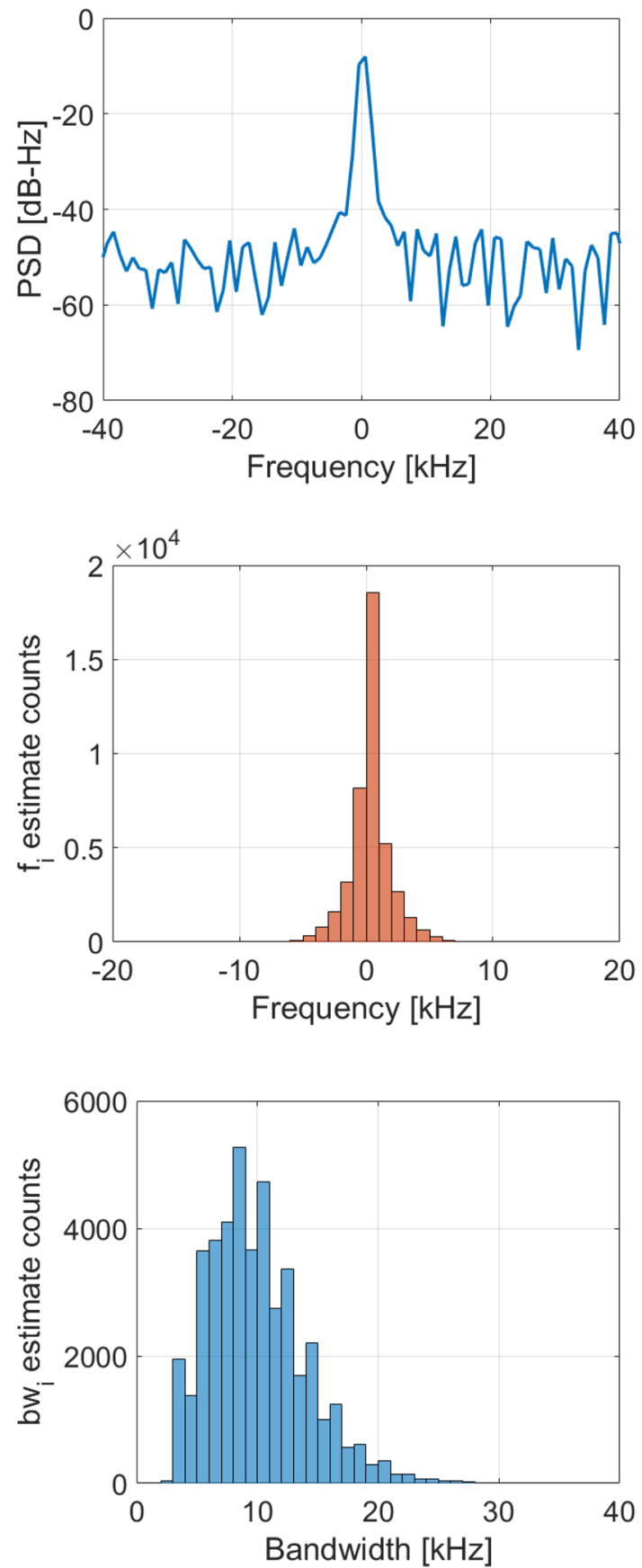

Fig. 10 NBI estimation performance. PSD of the signal affected by interference at $f_{\mathrm{L} 1 / \mathrm{E} 1}$ (top), distribution of the estimated $f_{i}$ (middle), and distribution of the estimated $b w_{i}$ (bottom)

For this reason, the rest of this section presents results that were obtained by using the threshold $T=\mu_{\mathrm{PSD}}+3 \cdot \sigma_{\mathrm{PSD}}$.

Every time when at least one interfered band is detected, estimation of the interference center frequency and bandwidth are carried out. A set of frequency estimates and a set of bandwidth estimates are, therefore, obtained in each test. The proposed technique was also evaluated in terms of its capability to compute such estimates. For the sake of simplicity, only the test case $\mathrm{i}$ is presented in Fig. 10.

In particular, a close-up view of the signal PSD in the interval $f_{\mathrm{L} 1 / \mathrm{E} 1} \pm 40 \mathrm{kHz}$ is given in the top panel, whereas the middle and the bottom panels show how many times each value of, respectively, the frequency estimates $\left(f_{i}\right)$ set and the bandwidth estimates $\left(b w_{i}\right)$ set has been obtained during the test. It can be observed that the estimated bandwidth values are distributed around the correct one $(\sim 9.5 \mathrm{kHz})$ and that the estimation of the center frequency is also quite accurate with errors in the range of only $\pm 5 \mathrm{kHz}$ from the true value.

The mitigation capability of the proposed method was assessed by investigating the improvement in the $C / N_{0}$ degradation when applying the mitigation technique. Each plot in Fig. 11 shows, for one interference profile, the average $C / N_{0}$ loss over time for GPS (blue lines) and Galileo (orange lines) when implementing (dashed lines) and when not implementing (solid lines) the technique.

As it can also be seen from Table 3, in all the cases, the $C / N_{0}$ drop caused by the interference is significantly reduced when the NBI countermeasure is applied.

The benefits of using the proposed NBI countermeasure were also assessed in the position domain. In the presence of NBI, the variance in the position solution increases. As an example, the horizontal $\left(\sigma_{\mathrm{h}}\right)$ and vertical $\left(\sigma_{\mathrm{v}}\right)$ standard deviations of the Javad multi-GNSS professional grade receiver's position solution in the absence and in the presence of interfering signal are provided in Table 4 for all the simulated cases. Single-constellation solutions were computed, and the deviation was considered with respect to the average solution in the absence of interference. For each case, the results related to the highly affected signal(s) only are presented. As it can be seen from Table 4, the impact of NBI on Javad's positioning solution in all the cases is clearly visible.

However, as it can be seen from Fig. 12, the presented NBI mitigation method significantly improves the position solution. In particular, Fig. 12 shows the east-north-up deviations of the FGI-GSRx solution for GPS and for Galileo in test iv when the mitigation is not applied (blue lines) and when it is applied (orange lines). In case of NBI unmitigated GPS-based position solution, namely the blue curves in the top panel of Fig. 12, few satellites were unable to pass the $C / N_{0}$ signal masking, i.e., $C / N_{0}>33 \mathrm{~dB}-\mathrm{Hz}$, of FGI-GSRx to be considered for computing position solution, which resulted in increased position error even after the interference was set off.

A significant improvement is obtained in all the tested cases, as shown in Table 5, which provides the values of $\sigma_{\mathrm{h}}$ and $\sigma_{\mathrm{v}}$ when applying and when not applying the countermeasure.

The position domain results appear to be consistent with those obtained in the tracking domain. For example, in the case of interference severely affecting both GPS and Galileo 
Fig. 11 Average GPS and Galileo $C / N_{0}$ loss when applying and not applying the proposed countermeasure for test case i (top left), test case ii (top right), test case iii (bottom left), and test case iv (bottom right)
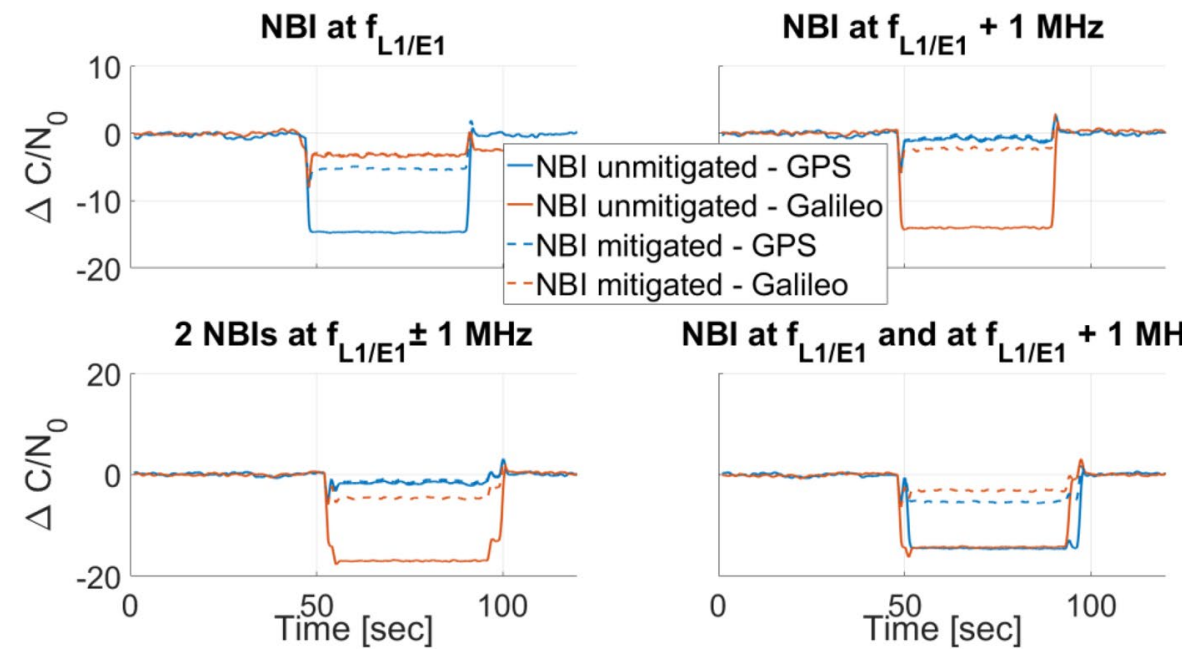

$\mathrm{NBI}$ at $\mathrm{f}_{\mathrm{L} 1 / \mathrm{E} 1}$ and at $\mathrm{f}_{\mathrm{L} 1 / \mathrm{E} 1}+1 \mathrm{MHz}$

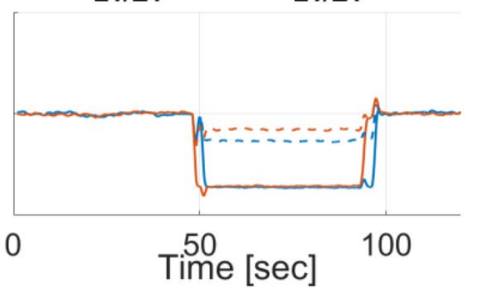

Table 3 Average $C / N_{0}$ gain with NBI countermeasure

\begin{tabular}{lll}
\hline Test case id & Highly affected signal(s) & $\begin{array}{l}\text { Average } \\
C / N_{0} \text { gain } \\
(\mathrm{dB})\end{array}$ \\
\hline i & GPS L1 C/A & $\sim 9.4$ \\
ii & Galileo E1 & $\sim 11.7$ \\
iii & Galileo E1 & $\sim 12.4$ \\
iv & GPS L1C/A & $\sim 9.1$ \\
& Galileo E1 & $\sim 11.2$ \\
\hline
\end{tabular}

Table 4 Horizontal and vertical standard deviation of Javad receiver

\begin{tabular}{|c|c|c|c|c|c|}
\hline \multirow[t]{2}{*}{ Test case id } & \multirow[t]{2}{*}{$\begin{array}{l}\text { Highly affected } \\
\text { signal(s) }\end{array}$} & \multicolumn{2}{|c|}{$\begin{array}{l}\text { Interference } \\
\text { off }\end{array}$} & \multicolumn{2}{|c|}{$\begin{array}{l}\text { Interference } \\
\text { on }\end{array}$} \\
\hline & & $\sigma_{\mathrm{h}}(\mathrm{m})$ & $\sigma_{\mathrm{v}}(\mathrm{m})$ & $\sigma_{\mathrm{h}}(\mathrm{m})$ & $\sigma_{\mathrm{v}}(\mathrm{m})$ \\
\hline $\mathrm{i}$ & GPS L1 C/A & 0.07 & 0.1 & 0.28 & 0.44 \\
\hline ii & Galileo E1 & 0.06 & 0.08 & 0.12 & 0.32 \\
\hline iii & Galileo E1 & 0.07 & 0.07 & 0.3 & 0.49 \\
\hline \multirow[t]{2}{*}{ iv } & GPS L1C/A & 0.07 & 0.11 & 0.18 & 0.28 \\
\hline & Galileo E1 & 0.07 & 0.1 & 0.28 & 0.47 \\
\hline
\end{tabular}

(case iv), the mitigation improvement in Galileo is higher than GPS.

In order to show more clearly the full adaptiveness of the method, an additional test was carried out. Similarly to the other cases, the interference source is turned on after an initial interference-free interval, but, in this case, the interference center frequency, bandwidth, and power vary over time, as described in Table 6. As it can be observed in Fig. 13, the proposed method is capable of coping with a time-varying NBI profile. For both GPS (top panel) and Galileo (bottom panel), the implementation of the NBI countermeasure (dashed lines) reduces
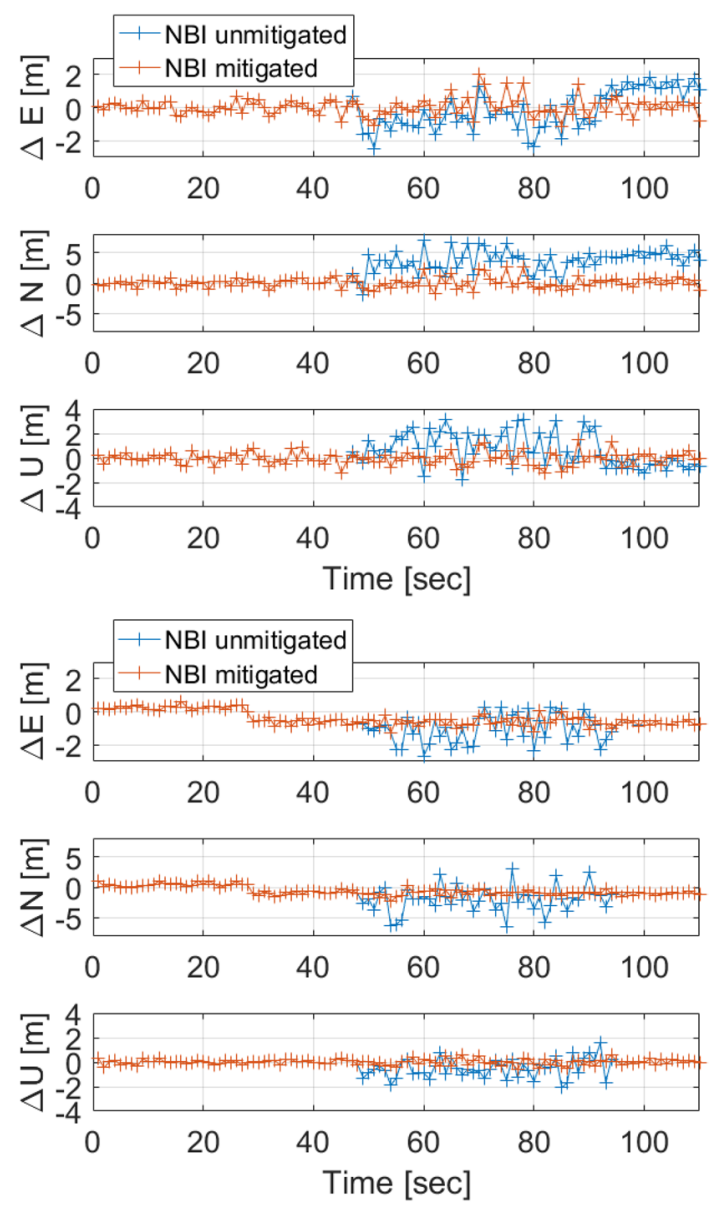

Fig. 12 East-north-up deviation in test case iv for GPS-based solution (top) and Galileo-based solution (bottom)

significantly the average $C / N_{0}$ loss which is caused by the interference when the mitigation is not applied (solid lines). The test duration is $2 \mathrm{~min}$ as in the other test cases, but the close-up view from the second 20th to 100th only 
Table 5 Horizontal and vertical standard deviation during the interference interval

\begin{tabular}{lllllll}
\hline Test case id & $\begin{array}{l}\text { Highly affected } \\
\text { signal(s) }\end{array}$ & \multicolumn{2}{l}{$\begin{array}{l}\text { No mitigation } \\
\text { applied }\end{array}$} & & \multicolumn{2}{l}{$\begin{array}{l}\text { Mitigation } \\
\text { applied }\end{array}$} \\
\cline { 3 - 4 } & & $\sigma_{\mathrm{h}}(\mathrm{m})$ & $\sigma_{\mathrm{v}}(\mathrm{m})$ & & $\sigma_{\mathrm{h}}(\mathrm{m})$ & $\sigma_{\mathrm{v}}(\mathrm{m})$ \\
\hline i & GPS L1 C/A & 1.8 & 1.38 & & 0.7 & 0.36 \\
ii & Galileo E1 & 2.19 & 0.55 & & 0.44 & 0.13 \\
iii & Galileo E1 & 2.72 & 0.86 & 0.51 & 0.17 \\
iv & GPS L1C/A & 1.54 & 0.92 & 0.83 & 0.37 \\
& Galileo E1 & 1.62 & 0.5 & 0.42 & 0.17 \\
\hline
\end{tabular}

is shown, to provide a clearer picture of the interference interval.

These results confirm that the proposed method is capable of coping with a scenario where narrowband interferers appear at different times in different portions of the GNSS spectrum, with different powers and different bandwidths.

\section{Comparison with adaptive notch filtering technique}

Finally, the implemented countermeasure was compared to the approach that has been extensively used by the GNSS community against NBI, and that is based on the adaptive notch filter described in Fig. 14. The input signal $x[n]$ is filtered by an auto-regressive moving average (ARMA) structure composed of three blocks: the auto-regressive (AR), the moving average (MA), and the adaptive block.

The MA and AR transfer functions are given by:

$H_{\mathrm{MA}}(z)=1-z_{0} z^{-1}$

$H_{\mathrm{AR}}(z)=\frac{1}{1-k z_{0} z^{-1}}$

In order to mitigate an interferer at $f_{i}$, one zero in $z_{0}=\alpha e^{-j 2 \pi f_{i}}$ is required and, at the same time, a pole in $z=k z_{0}$ is required to compensate for the effects of the zero.
The adaptive block is based on a normalized least mean square (LMS) algorithm (Haykin 2001) which iteratively minimizes the cost function:

$J[n]=E\left\{\left|x_{f}[n]\right|^{2}\right\}$,

where $x_{f}[n]$ is the output of the filter.

The minimization is performed with respect to the complex parameter $z_{0}$ using the iterative rule:

$z_{0}[n+1]=z_{0}[n]-\mu[n] \cdot g(J[n])$,

where

$g(J[n])=\nabla_{z_{0}}\left\{\left|x_{f}[n]\right|^{2}\right\}=-4 x_{f}[n] \cdot\left(x_{i}^{*}[n-1]\right)$

is the stochastic gradient of the cost function, while

$\mu[n]=\frac{\delta}{E_{x_{i}[n]}}$

is the algorithm step. $E_{x_{i}[n]}$ is an estimate of $E\left\{\left|x_{i}[n]\right|^{2}\right\}$, with $x_{i}[n]$ being the output of the AR block, and $\delta$ is the nonnormalized LMS algorithm step, which controls the convergence properties of the algorithm.

The results presented here are obtained for a case with one interferer at $f_{\mathrm{L} 1 / \mathrm{E} 1}$ and one at $f_{\mathrm{L} 1 / \mathrm{E} 1}+1 \mathrm{MHz}$. The interfering signal was turned on at around 46th second after the start of the data capture that lasted, in this case, $60 \mathrm{~s}$. The detection/estimation unit is used to determine if mitigation is required and to estimate frequencies and widths of the interfered bands. In one case, the proposed method is applied; hence, the mitigation is performed by applying one notch filter for each identified interfered band. The parameter $k$, which is derived from the estimated bandwidth, and the estimated frequency are used to configure the filter which processes all the signal samples in a $T_{\text {coh }}$ long block. In the other case, the estimation of the bandwidths is not exploited, while the frequencies estimated by the detection/estimation block are used to only initialize the zero $z_{0}$ of a corresponding number of adaptive notch filters. Each filter processes the
Table 6 Time-varying interference profile

\begin{tabular}{|c|c|c|c|c|}
\hline Center frequency & Bandwidth (kHz) & $\begin{array}{c}\text { Interference/GNSS signal } \\
\text { power ratio }(\mathrm{dB})\end{array}$ & 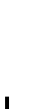 & \\
\hline$f_{\mathrm{L} 1 / \mathrm{E} 1}$ & 90 & 25 & & NBI start time \\
\hline$f_{\mathrm{L} 1 / \mathrm{E} 1}+0.5 \mathrm{MHz}$ & 180 & 35 & & $5 \mathrm{~s}$ \\
\hline$f_{\mathrm{L} 1 / \mathrm{E} 1}-1 \mathrm{MHz}$ & 90 & 20 & & $10 \mathrm{~s}$ \\
\hline$f_{\mathrm{L} 1 / \mathrm{E} 1}+1.5 \mathrm{MHz}$ & 200 & 35 & & \\
\hline$f_{\mathrm{L} 1 / \mathrm{E} 1}-1.2 \mathrm{MHz}$ & 200 & 15 & & $20 \mathrm{~s}$ \\
\hline$f_{\mathrm{L} 1 / \mathrm{E} 1}-0.5 \mathrm{MHz}$ & 180 & 30 & $\downarrow$ & NBI end time \\
\hline
\end{tabular}



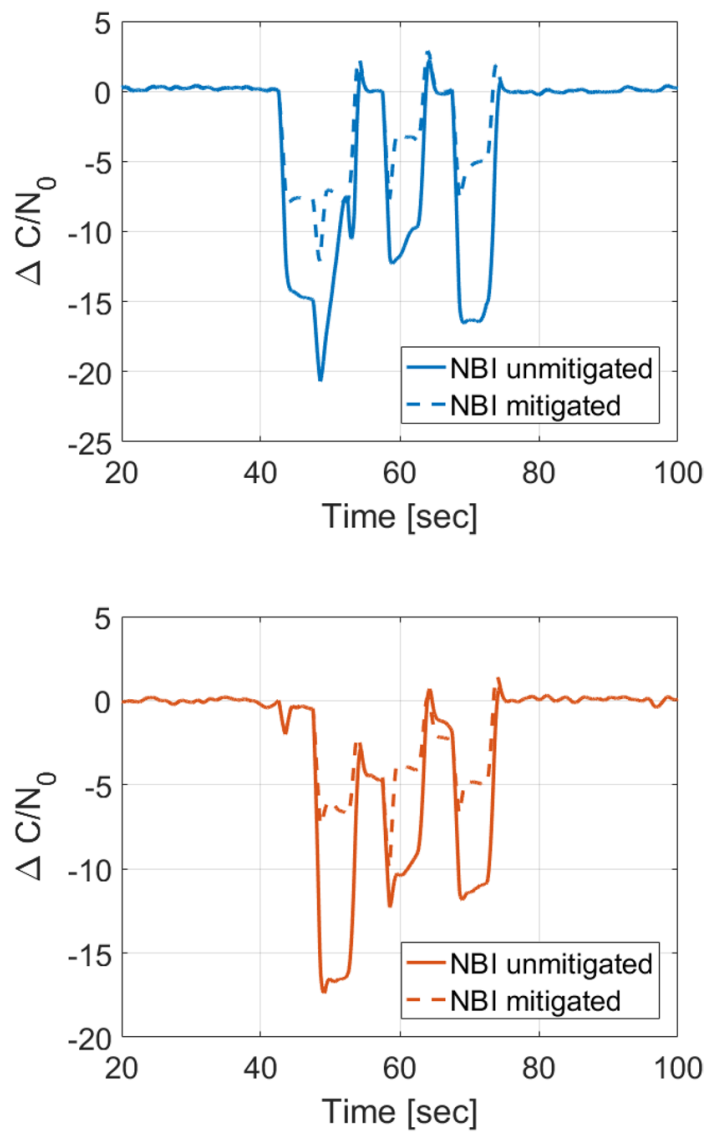

Fig. 13 Average $C / N_{0}$ loss for GPS (top) and Galileo (bottom) when applying and not applying the proposed countermeasure in the timevarying NBI test case

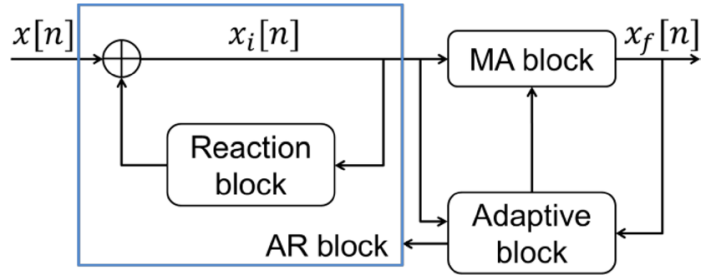

Fig. 14 Adaptive notch filter structure

$T_{\text {coh }}$ long block of IF samples by using a fixed $k$ value and by progressively adapting $z_{0}$, hence, the notch frequency, using the LMS approach described above. In this case, the notch frequency adaptation is done sample by sample. Two values of $k$ were tested, $k=0.95$ and $k=0.985$.

It can be observed, in Fig. 15, that the proposed approach (orange line) is capable of gaining the average $C / N_{0}$ loss by around $9 \mathrm{~dB}$ for GPS, as shown in the top panel, and by around $12 \mathrm{~dB}$ for Galileo, as shown in the bottom panel, whereas the performance of the adaptive notch filter-based approach depends on the value of $k$. For $k=0.985$ (purple line), the $C / N_{0}$ degradation is improved by around $2 \mathrm{~dB}$ for
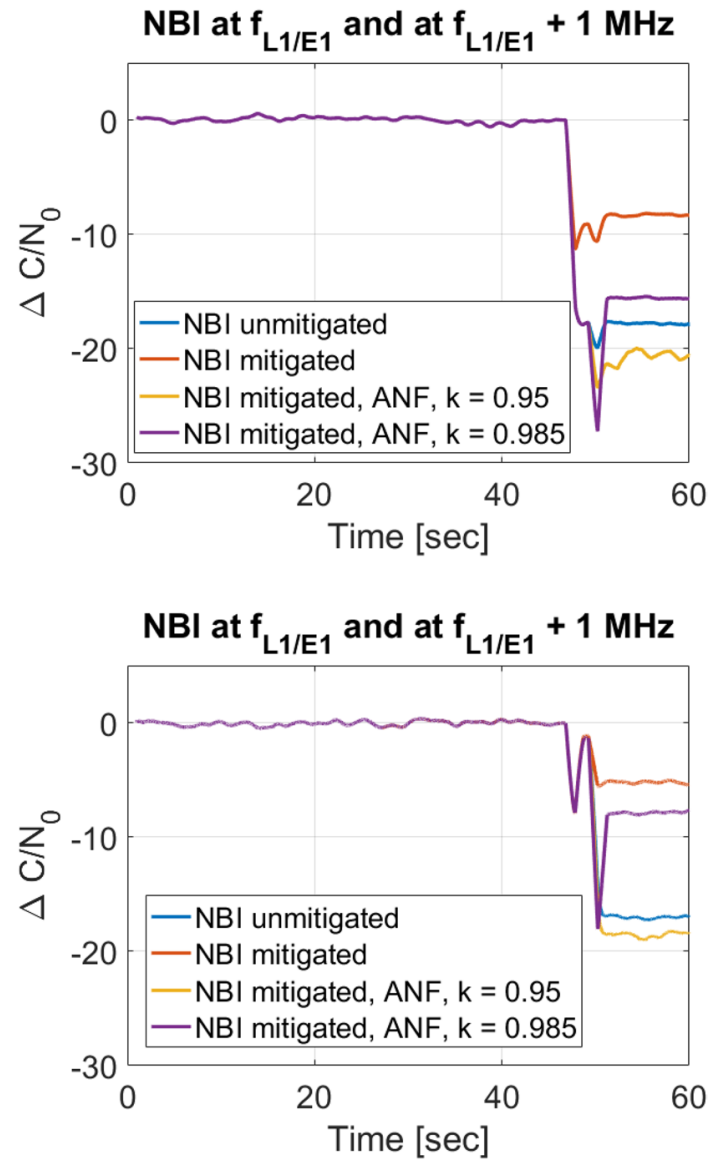

Fig. 15 Average $C / N_{0}$ loss for GPS (top) and for Galileo (bottom)

GPS and by around $10 \mathrm{~dB}$ for Galileo, which is, however, less than with the proposed implementation, but for $k=0.95$ (yellow line), the performance is even worse than when no mitigation is applied. This is due to the fact that when $k$ is equal to 0.985 , the notch width is narrow enough to be able to track one of the two interfered frequencies at a time, but when $k$ is equal to 0.95 , the notch width is wider and not able to track one interfered frequency only at a time. Instead, the filter progressively diverges away from the first interfered band and ends up tracking the frequency in the middle between the two interference spikes.

Even in the simpler cases of single NBI, the mitigation performance depends on the value of $k$. Experimental results showed that a too wide notch can degrade the $C / N_{0}$, even for the GNSS signal that is originally not much affected by the interference. Having a fully adaptive mitigation scheme is, therefore, fundamental. Since the number of interferers that may appear in the GNSS band, their center frequency and their bandwidth cannot be known a priori, it is of utmost importance to use a totally flexible mitigation scheme capable of adapting the number of notch filters to be applied, their notch frequency, and their notch width. 
The importance of adapting the notch width in order to optimize the NBI suppression has also been underlined in Nguyen et al. (2014), where the authors present a technique addressing the issue. However, the approach described in there presents some limitations that our method overcomes. First, it tackles single NBI only, while our technique is capable of coping with any number of narrow interfered bands in the GNSS spectrum. Second, the time needed from the bandwidth estimation algorithm to converge to the steadystate increases with the interference bandwidth, while in our case the time needed to estimate the NBI bandwidth does not have any dependency on the interference width. Third, the technique has a limit related to the selection of the prefixed width of the two adaptive notch filters used for the bandwidth estimation: it is able to finely estimate the bandwidth only when the width of the notch filters' band is less than half of the NBI width.

\section{Conclusions}

The issue of unintentional NBI, which represents one of the main threats to GNSS-based applications, has mostly been addressed with the use of the adaptive notch filtering technique capable of estimating the interference frequency. However, the center frequency is not the only unknown parameter of the interference, since also the number of interfered bands and their width cannot be known a priori. Therefore, a higher level of adaptability is required to cope with the unpredictable and diverse nature of unintentional interfering events.

We presented a new fully adaptive solution against unintentional NBI. The proposed technique was evaluated in terms of detection, estimation, and mitigation capability, and simulation results showed that it is capable of promptly detecting the NBI event, accurately estimating the interfered center frequencies and bandwidths, and efficiently mitigating the NBI effect on the signal. A comparison with the adaptive notch filtering technique proved the importance of having a fully adaptive countermeasure. Moreover, an analysis of the impact of different NBI profiles on GPS L1 C/A and Galileo E1 was provided. Future work includes analysis of the intentional jamming impact on multi-GNSS receivers and investigation of the proposed solution in the presence of such intentional interference sources.

Acknowledgements This research has been conducted within the project entitled "Information Security of Location Estimation and Navigation Applications (INSURE)". The INSURE project is funded by the Academy of Finland under the Grant no. 303576. This work is also partially supported by the EU H2020 project entitled "Standardization of GNSS Threat reporting and Receiver testing through International Knowledge Exchange, Experimentation and Exploitation (STRIKE3)".
Open Access This article is distributed under the terms of the Creative Commons Attribution 4.0 International License (http://creativeco mmons.org/licenses/by/4.0/), which permits unrestricted use, distribution, and reproduction in any medium, provided you give appropriate credit to the original author(s) and the source, provide a link to the Creative Commons license, and indicate if changes were made.

\section{References}

Axell E, Eklöf FM, Alexandersson M, Johansson M, Akos DM (2015) Jamming detection in GNSS receivers: performance evaluation of field trials. Navigation 61(1):73-82

Balaei A, Dempster A (2009) A statistical inference technique for GPS interference detection. IEEE Trans Aerosp Electron Syst 45(4):1499-1511

Bhuiyan MZH, Kuusniemi H, Söderholm S, Airos E (2014a) The impact of interference on GNSS receiver observables-a running digital sum based simple jammer detector. Radioeng $\mathbf{J}$ 23(3):898-906

Bhuiyan MZH, Söderholm S, Thombre S, Ruotsalainen L, Jaakkola MK, Kuusniemi H (2014b) Performance evaluation of carrierto-noise density ratio estimation techniques for BeiDou B1 signal. In: Proceedings of ubiquitous positioning, indoor navigation and location-based services conference, Corpus Christi, Texas, USA, November 20-21, pp 19-25

Bhuiyan MZH, Söderholm S, Thombre S, Ruotsalainen L, Kuusniemi $\mathrm{H}(2014 \mathrm{c})$ Overcoming the challenges of BeiDou receiver implementation. Sensors 14(11):22082-22098

Borio D, Camoriano L, Mulassano P (2006) Analysis of the one-pole notch filter for interference mitigation-Wiener solution and loss estimations. In: Proceedings of ION GNSS 2006, Institute of Navigation, Fort Worth, Texas, USA, September 26-29, pp $1849-1860$

Borio D, Dovis F, Kuusniemi H, Lo Presti L (2016) Impact and detection of GNSS jammers on consumer grade satellite navigation receivers. Proc IEEE 104(6):1233-1245

Calcagno R, Fazio S, Savasta S, Dovis F (2010) An interference detection algorithm for COTS GNSS receivers. In: Proceedings of ESA workshop on satellite navigation technologies and European workshop on GNSS signals and signal processing (NAVITEC), Noordwijk, The Netherlands, December 8-10, pp 1-8

Daneshmand S, Broumandan A, Nielsen J, Lachapell G (2013) Interference and multipath mitigation utilising a two-stage beamformer for global navigation satellite systems applications. IET Radar Sonar Navig 7(1):55-66

Dovis F, Musumeci L (2016) Use of the Karhunen-Loève transform for interference detection and mitigation in GNSS. ICT Express 2(1):33-36

Dovis F, Musumeci L, Linty N, Pini M (2012) Recent trends in interference mitigation and spoofing detection. Int J Embed Real Time Commun Syst 3(3):1-17

European Union (2016) European GNSS (Galileo) open service signal-in-space interface control document. OS SIS ICD, issue 1.3, December. https://www.gsc-europa.eu/system/files/galil eo_documents/Galileo-OS-SIS-ICD.pdf. Accessed 18 July 2018

Fadaei N, Jafarnia-Jahromi A, Lachapelle G (2015) Detection, characterization and mitigation of GNSS jammers using windowedHHT. In: Proceedings of ION GNSS + 2015, Institute of Navigation, Tampa, Florida, USA, September 14-18, pp 1625-1633

Fraunhofer Institute for Integrated Circuits IIS (2013) G-TEC RFFE (TeleOrbit) datasheet. https://www.iis.fraunhofer.de/content/ 
dam/iis/de/doc/lv/los/lokalisierung/SatNAV/TO_DataSheet_ GTEC_RFFE_2013-02-20.pdf. Accessed 18 July 2018

Global Positioning Systems Directorate (2015) NAVSTAR GPS space segment/navigation user segment interfaces. IS-GPS-200, December. https://www.gps.gov/technical/icwg/IRN-IS200H-001+002+003_rollup.pdf. Accessed 18 July 2018

Haykin S (2001) Adaptive filter theory, 4th ed. Prentice Hall, Upper Saddle River

Kuusniemi H, Airos E, Bhuiyan MZH, Kröger T (2012) GNSS jammers: how vulnerable are consumer grade satellite navigation receivers? Eur J Navig 10(2):14-21

Lindstrom J, Akos D, Isoz O, Junered M (2007) GNSS interference detection and localization using a network of low cost frontend modules. In: Proceedings of ION GNSS 2007, Institute of Navigation, Fort Worth, Texas, USA, September 25-28, pp $1165-1172$

Motella B, Lo Presti L (2014) Methods of goodness of fit for GNSS interference detection. IEEE Trans Aerosp Electron Syst 50(3): 1690-1700

Musumeci L, Dovis F (2014) Use of the wavelet transform for interference detection and mitigation in global navigation satellite systems. Int J Navig Obs 2014:262186

Nguyen TT, The VL, Ta TH, Nguyen H-LT, Motella B (2014) An adaptive bandwidth notch filter for GNSS narrowband interference mitigation. REV J Electron Commun 4(3-4):59-68

Parkinson BW, Spilker J Jr, Axelrad P, Enge P (1996) Global positioning system: theory and applications, vol I. AIAA, Washington, DC

Raasakka J, Orejas M (2014) Analysis of notch filtering methods for narrowband interference mitigation. In: Proceedings of IEEE/ION PLANS 2014, Institute of Navigation, Monterey, California, USA, May 5-8, pp 1282-1292

Ruotsalainen L, Kuusniemi H, Bhuiyan MZH, Chen L, Chen R (2012) A two dimensional pedestrian navigation solution aided with a visual gyroscope and a visual odometer. GPS Solut 17(4):575586. https://doi.org/10.1007/s10291-012-0302-8

Ruotsalainen L, Kirkko-Jaakkola M, Bhuiyan MZH, Söderholm S, Thombre S, Kuusniemi H (2014) Deeply-coupled GNSS, INS and visual sensor integration for interference mitigation. In: Proceedings of ION GNSS + 2014, Institute of Navigation, Tampa, Florida, September 8-12, pp 2243-2249

Sánchez-Naranjo SM, Ferrara NG, Pásnikowski MJ, Raasakka J, Shytermeja E, Ramos-Pollán R, González Osorio FA, Martínez D, Lohan ES, Nurmi J, Toledo López M, Kotaba O, Julien O (2017) GNSS vulnerabilities. Multi-technology positioning. Springer, Cham, pp 55-77

Sheridan K, Ying Y, Whitworth T (2012) Pre- and post-correlation GNSS interference detection within software defined radio. In: Proceedings ION GNSS 2012, Institute of Navigation, Nashville, Tennessee, USA, September 17-21, pp 3542-3548

Söderholm S, Bhuiyan MZH, Thombre S, Ruotsalainen L, Kuusniemi H (2016) A multi-GNSS software-defined receiver: design, implementation, and performance benefits. Ann Telecommun 71(78):399-410 August.

Spectracom (2017) GSG-5/6 series GNSS simulator user manual with SCPI guide. Spectracom Part No.: 4031-600-54001, Revision: 25, May 2017. https://spectracom.com/sites/default/files/documentfiles/GSG_UserManual_PN4031-600-54001_R25.pdf

STRIKE3 (2016) Standardisation of GNSS threat reporting and receiver testing through international knowledge exchange, experimentation and exploitation [STRIKE3]. http://www.gnssstrike3.eu/. Accessed 18 July 2018

Thombre S, Bhuiyan MZH, Söderholm S, Kirkko-Jaakkola M, Ruotsalainen L, Kuusniemi H (2015) A software multi-GNSS receiver implementation for the Indian Regional Navigation Satellite System. IETE J Res 62(2):246-256 October.
Thombre S, Bhuiyan MZH, Eliardsson P, Gabrielsson B, Pattinson M, Dumville M, Fryganiotis D, Hill S, Manikundalam V, Pölöskey M, Lee S, Ruotsalainen L, Söderholm S, Kuusniemi H (2018) GNSS threat monitoring and reporting: past, present, and a proposed future. R Inst Navig J 71(3):513-529

Wildemeersch M, Cano Pons E, Rabbachin A, Fortuny Guasch J (2010) Impact study of unintentional interference on GNSS receivers. Tech. Rep. of the EC Joint Research Center, Security Technology Assessment Unit, EUR 24742 EN

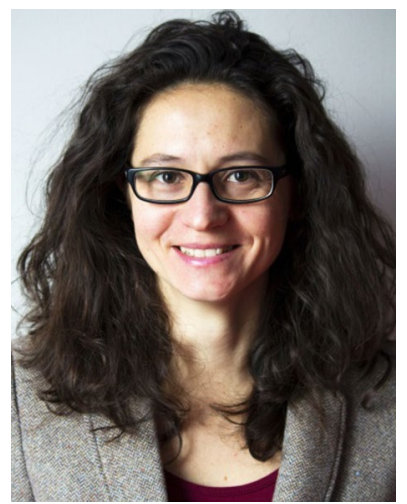

Nunzia Giorgia Ferrara is a research scientist in the Department of Navigation and Positioning at the Finnish Geospatial Research Institute, and she is also a $\mathrm{Ph} . \mathrm{D}$. candidate at the Electronics and Communications Engineering Department of Tampere University of Technology where she was a Marie Curie Fellow from 2014 to 2016. Her research focuses on multi-GNSS signals and interference detection and mitigation

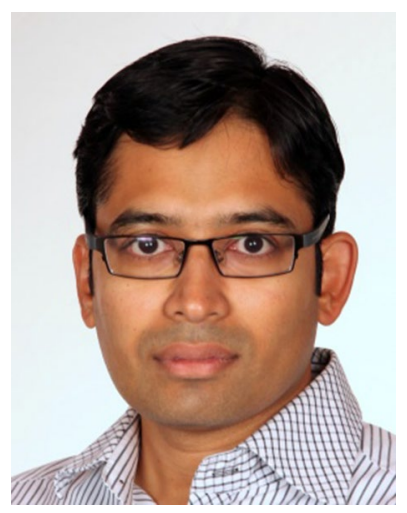

Mohammad Zahidul H. Bhuiyan is currently working in the Department of Navigation and Positioning at the Finnish Geospatial Research Institute as a Research Manager with research interests covering various aspects of GNSS receiver design, GNSS vulnerabilities detection and mitigation, integration of GNSS with different sensors, etc. $\mathrm{He}$ is also acting as the deputy head of the 'Satellite and Radio Navigation' research group of the institute

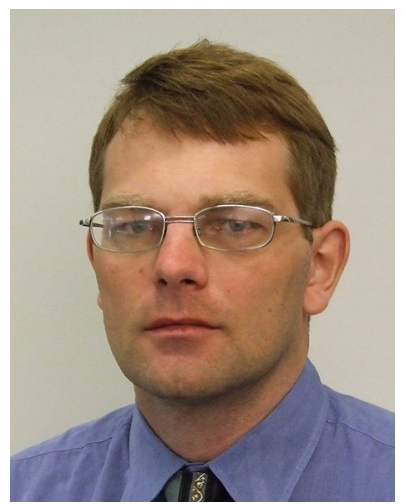

Stefan Söderholm received his M.Sc. degree from Åbo Akademi University, Department of Experimental Physics, in 1991 and his Licentiate degree from University of Turku, Department of Applied Physics, in 1996. He joined the Department of Navigation and Positioning at the Finnish Geospatial Research Institute, in September 2013 and is now working as a research group leader with research interests covering multi-GNSS receiver design, BOC signal processing, GNSS interference 


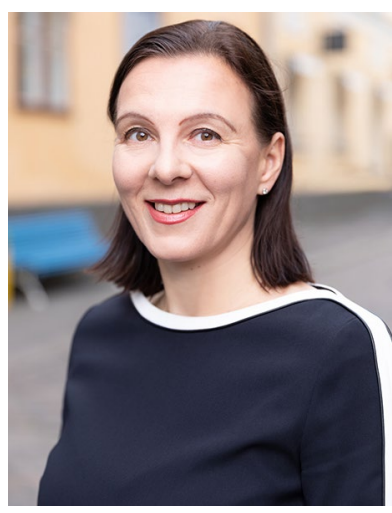

Laura Ruotsalainen is a Research Manager and Deputy Director of the Department of Navigation and Positioning at the Finnish Geospatial Research Institute, Finland, where she leads a research group on Sensors and indoor navigation. Her current research interests cover visionaided navigation, adaptive integration of sensors and radio positioning means for robust navigation, situational awareness and GNSS interference mitigation

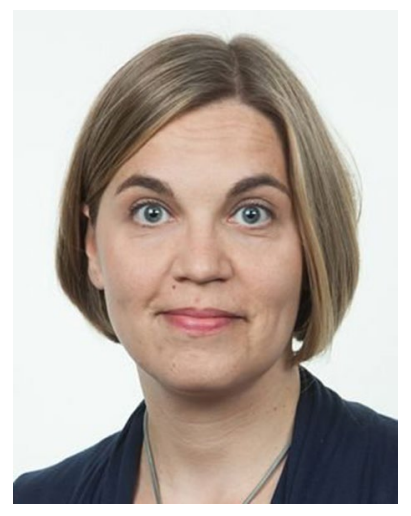

Heidi Kuusniemi is the Director of the Department of Navigation and Positioning at the Finnish Geospatial Research Institute. She is also an Adjunct Professor at Aalto University, Department of Built Environment, and at the Tampere University of Technology, Department of Electronics and Communications Engineering, Finland. She is the President of the Nordic Institute of Navigation 